\title{
Research on Underwater Polarization Image Segmentation Inspired by Biological Optic Nerve
}

\author{
Huibin Wang, Yurong Wu, Jie Shen, Zhe Chen \\ College of Computer and Information Engineering \\ Hohai University \\ Nanjing, China \\ E-mail: hbwang@hhu.edu.cn
}

\begin{abstract}
Due to effects of the light by water and other particles, the quality of underwater image will degrade. The traditional underwater image segmentation methods based on intensity and spectrum have difficulty in determining boundary. Inspired by the visual system of mantis shrimps, this paper constructed a feedback neural network model, in which the parameters were optimized using machine learning method. Based on this model, we combine the polarization and intensity information to achieve the underwater polarization image segmentation. The results of experiment prove that the neural network model designed in this paper can improve the accuracy of underwater image segmentation.
\end{abstract}

Keywords-underwater polarization image segmentation; optic nerve of mantis shrimps; feedback neural network model; parameters optimization

\section{INTRODUCTION}

Due to absorption and scattering of the light by water and other particles, underwater images have drawbacks of low contrast, blur edge and serious noise [1]. These drawbacks result in inaccurate boundary and edge position. Therefore, the research of image segmentation technology has been become a new focus in the field of underwater image processing in recent years

The light intensity and spectrum in water vary strongly and serious attenuation with depth, so it is difficult to determine the target's boundary and edge only depending on the light intensity and spectrum of image. While the polarization feature of light is much more predictable and stable [2]. And polarization imaging can filter out background scatting light interference. Consequently, it can improve the image contrast. But there are problems: when the background light is eliminated, the reflected light of target is also largely filtered out, which would affect the image quality. Whether we can combine polarization and light intensity information to apply to underwater image segmentation? In recent years, research results in the field of biology show that: in the ocean, the visual system of mantis shrimps has a great ability in processing light intensity, spectral and polarization information. It can effectively use these information to detect and identify targets in highly scattering of the water [3] .On the one hand, this visual system demonstrates that it is feasible to combine light intensity and polarization information. On the other hand, it would provide a technical approach to utilize of these information.

In order to combine light intensity and polarization information used for image segmentation, this paper designed a neural network model inspired by the visual system of mantis shrimps, then constructed a frame of underwater polarization image segmentation based on this model. Through the polarization and light intensity information fusion processing, the accuracy of underwater image segmentation can be effectively improved.

\section{Model Design OF NeURAL NeTwORK}

\section{A. Visual Structure Feature of Mantis Shrimps}

The research of biology in recent years can be summarized as follow: the optic nerve system of mantis shrimps is divided into two levels by central cartridges cell. It makes mantis shrimps tackle the obtained visual information by a classification processing way [4]. The lower-layer consists of cartridge cells, which are information processing array. In this layer, the information processing array coupling the optical information acquired by photoreceptors, and extract the primary information (such as polarization, spectral, light intensity and spatial information). In high layer, the neurons process the information extracted by low neurons, and fuse the results of the processing. Also, the visual fibers of eight photoreceptors in one ommatidium terminate in different levels of the cartridges. In geometry, it indicates that the polarization, intensity and spectral information are processed in separate channels [5]. From above, parallel transmission and classification processing the acquired optical information are the important characteristics of the visual system of mantis shrimps. The advantage of these characteristics can be concluded as follow: parallel transmission can increase signal transmission bandwidth and prevent the information from mutual interference, which avoid the loss of information. And this hierarchical processing pattern is possible to reduce the time cost for information processing in high order neurons.

\section{B. Model Design of Feedback Neural Network \\ I) Information processing mechanism of mantis shrimps}

In the optic system of mantis shrimps, the different information of characterization target that extracted by low order neurons are inputs of high order neurons. The 
information can make high order neurons generate different degree responses. Only when the stimulus reaches its own threshold, it would make the high order neurons excited. Then the neurons would be fired and generate impulse; otherwise, the neurons would be in resting state [6]. It also can be given by:

$$
H_{i}(t)= \begin{cases}1 & C_{i}(t)>s_{i} \\ 0 & \text { otherwise }\end{cases}
$$

where $C_{i}(t)$ is the stimulus of information $i$ at the instant $t$, $s_{i}$ is the stimulus threshold, $H_{i}(t)$ is the response impulse generated by neuron at the instant $t$ stimulated by information $i$.

In addition, if the different information from different channels arrive at the neuron within a short interval of time, the neuron would be a summation neuron [6]. It also can be given by:

$$
P\left(t_{i}\right)= \begin{cases}1 & t_{i}-t_{i-1}<T \\ 0 & \text { otherwise }\end{cases}
$$

where $t_{i}$ and $t_{i-1}$ is the instant of arrival of different information at the neuron, $T$ is the interval of time, $P\left(t_{i}\right)$ is the final response impulse generated at the instant $t_{i}$ by neuron which is stimulated by different information.

II) Model design of feedback neural network

Imitating the structure characteristics of visual system of mantis shrimps, this paper designed a multi-input singleoutput two layers neural network model, which can parallel process diversified information and fuse different information processing results. The first level is responsible for extracting the primary information, and the second is used for processing primary information and fusing the processing results. Neuron is the basic unit of neural network model, which is a summation neuron. It consists of two parallel channels that used for processing different information, fusing the different information processing results and forming the final result. The structure of neuron $j$ is shown in Fig. 1.

The structure of neuron $j$ includes three parts: input part, impulse generation part and fusing part. Each part is described as follow.

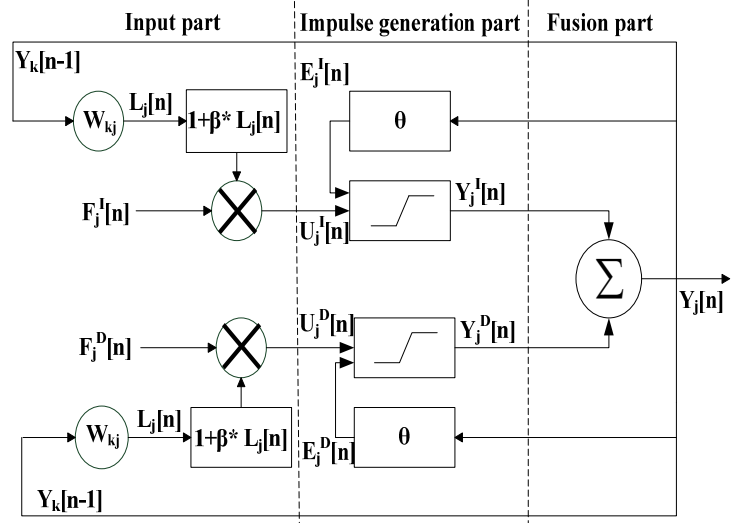

Figure 1. Structure of neuron $j$
Neuron $j$ consists of two channels. The internal stimulus of each channel is respectively $U_{j}^{I}[n]$ and $U_{j}^{D}[n]$, which are given by:

$$
\begin{aligned}
U_{j}^{I}[n] & =F_{j}^{I}[n]\left(1+\beta L_{j}[n]\right) \\
U_{j}^{D}[n] & =F_{j}^{D}[n]\left(1+\beta L_{j}[n]\right)
\end{aligned}
$$

where $F_{j}^{I}[n]$ and $F_{j}^{D}[n]$ are the current iteration external stimulus of neuron $j, \beta$ is the linking parameter presenting influence factor that is the total feedback of neighborhood neurons to the current iteration inputs, $n$ is the current iteration, i.e. discrete time step, $L_{j}[n]$ is the total feedback of neighborhood neurons, which is given by:

$$
L_{j}[n]=\sum W_{k j} Y_{k}[n-1]
$$

where $W_{k j}$ is the synaptic linking weigh between neuron $j$ and the neighborhood neuron $k, Y_{k}[n-1]$ is the last iteration output of neighborhood neuron $k$.

- Impulse generation part

According to the analysis of section II, when the stimulus reaches its own threshold, it would make the neurons excited. Then the neurons would be fired and generate impulse; otherwise, the neurons would be in resting state. Thus, according to the equation (1), the outputs of each channel are given by:

$$
\begin{array}{r}
Y_{j}^{I}[n]= \begin{cases}1, & U_{j}^{I}[n] \geq E_{j}^{I}[n] \\
0, & U_{j}^{I}[n]<E_{j}^{I}[n]\end{cases} \\
Y_{j}^{D}[n]= \begin{cases}1, & U_{j}^{D}[n] \geq E_{j}^{D}[n] \\
0, & U_{j}^{D}[n]<E_{j}^{D}[n]\end{cases}
\end{array}
$$

where $E_{j}^{I}[n]$ and $E_{j}^{D}[n]$ are the current iteration threshold of each channel, which are given by:

$$
\begin{aligned}
& E_{j}^{I}[n]=e^{-\alpha_{1}} E_{j}^{I}[n-1]+V_{E} Y_{j}[n-1] \\
& E_{j}^{D}[n]=e^{-\alpha_{2}} E_{j}^{D}[n-1]+V_{E} Y_{j}[n-1]
\end{aligned}
$$

where $\alpha_{1}$ and $\alpha_{2}$ are the time constant presenting the speed of threshold change, $E_{j}^{I}[n-1]$ and $E_{j}^{D}[n-1]$ are the last iteration threshold of each channel, $V_{E}$ is the amplitude coefficient of threshold regulator presenting the initial amplitude of threshold, $Y_{j}[n-1]$ is the last iteration output of neuron $j$.

- Fusion part

According to the analysis of section, when the stimulus reaches its own threshold, it would make the neurons excited. Then the neurons would be fired and generate impulse; otherwise, the neurons would be in resting state. Thus, according to the equation (1), the outputs of each channel are given by:

$$
Y_{j}[n]= \begin{cases}1, & \frac{1}{2} Y_{j}^{I}[n]+\frac{1}{2} Y_{j}^{D}[n] \geq 0.5 \\ 0, & \frac{1}{2} Y_{j}^{I}[n]+\frac{1}{2} Y_{j}^{D}[n]<0.5\end{cases}
$$

The above model is a single neuron processing information model. When all the neurons connect together, it would form a two-dimensional feedback neural network. 


\section{UNDERWATER IMAGE SEGMENTATION BASED ON THE FEEDBACK NEURAL NETWORK}

Image segmentation is the problem of partitioning an image into its constituent components. In wisely choosing a partition that highlights the role and salient properties of each component, we obtain a compact representation of an image [7]. The same region has similar attribute, and the adjacent region has different attribute. In the neural network model, when the external stimulus is exceed the threshold in each of channels, it would make the neuron excited. Then the neurons would be fired and generate impulse, at the same time, it would affect the inputs of adjacent neurons, through the feedback. The effect would make the stimulus of adjacent neurons rapidly lifting. When the stimulus reaches its threshold, it would make the adjacent neurons be fired and generate impulse. Moreover, as long as one of two channels can make neuron fire, the neuron would be fired and generate impulse. Therefore, this model can not only make some similar feature vector fire at the same time and different feature vector fire in different time, i.e. this characteristic can divide the image into different areas; but also deal with different information, through the cooperation finishing the analysis of scene. Based on above idea, this paper constructed a frame of underwater image segmentation shown in Fig. 2.

The underwater polarization image segmentation procedure is: capture 0 degree, 45 degree and 90 degree underwater target polarization image. Then, extract the images of total intensity and linear degree of polarization, put them as the inputs of the neural network model into two parallel channels, and process them based on the above designed feedback neural network model to achieve the underwater image segmentation. In the segmentation system, this paper exploits machine learning method to optimize the key parameters of neural network model. Because the differences between the information of target, as well as their changes in different conditions, especially in complex underwater environment. How to make sure the ability of information in characterization of target attributes, so that adaptively adjust the parameters of different channels to help to underwater target detection.

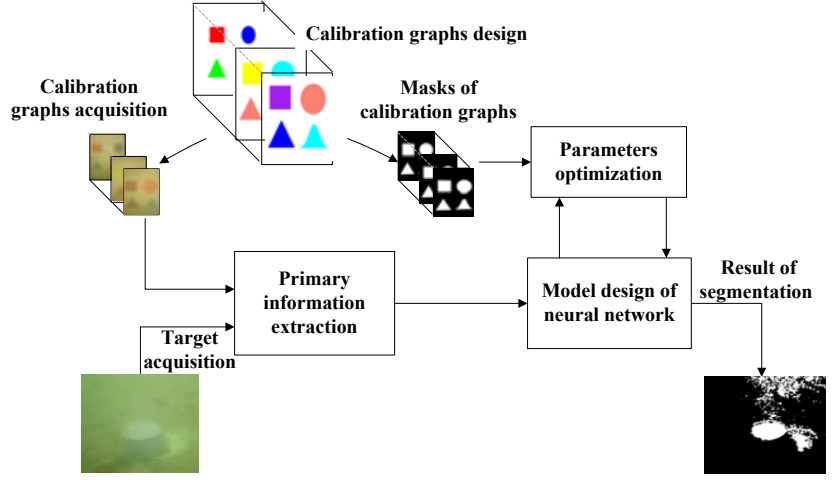

Figure 2. Frame of underwater image segmentation

\section{A. Primary Information Extraction}

In general, the polarization state of light is described by Stokes vector, which was put forward by G.G.S tokes in 1852 when he studied partially polarized light. Stokes parameter expression is given as follows:

$$
S=\left[\begin{array}{l}
S_{0} \\
S_{1} \\
S_{2} \\
S_{3}
\end{array}\right]=\left[\begin{array}{c}
I \\
Q \\
U \\
V
\end{array}\right]=\left[\begin{array}{c}
I_{0}+I_{90} \\
I_{0}-I_{90} \\
2 * I_{45}-I_{0}-I_{45} \\
I_{R C P}-I_{L C P}
\end{array}\right]
$$

where the parameter $I$ is the total intensity of the light, the parameter $Q$ is the amount of linear horizontal or vertical polarization, the parameter $U$ is the amount of linear 45 direction polarization, the parameter $V$ is the amount of right or left circular polarization contained within the beam. In most case, the parameter $V$ is regarded as zero. In addition, there are two important parameters, which are the linear degree of polarization (DOLP) and angle of polarization (AOP), which are given by:

$$
\begin{gathered}
D O L P=\frac{\sqrt{Q^{2}+U^{2}}}{I} \\
A O P=\frac{1}{2} \tan ^{-1} \frac{U}{Q}
\end{gathered}
$$

\section{B. Parameters of Neural Network Model Optimization}

Based on the design of this paper, the best image segmentation result is to find the optimum ignition time matrix. While in the neural network model the ignition time matrix of two channels have a tight relationship with the parameters of each channel. From the mathematical expression of the model we can conclude that it is critical to select the threshold in this model. If the change of threshold is too big, it would cause the image over-segmentation easily; similarly, if the change is too small, it would make the image under-segmentation. The adjustment of the threshold mainly depends on the regulation of parameters $\alpha_{1}$ and $\alpha_{2}$. Therefore, this paper optimize the parameter $\alpha_{1}$ and $\alpha_{2}$ that have a great effect on image segmentation results through the machine learning method, so that make the designed neural network model be in an optimal state.

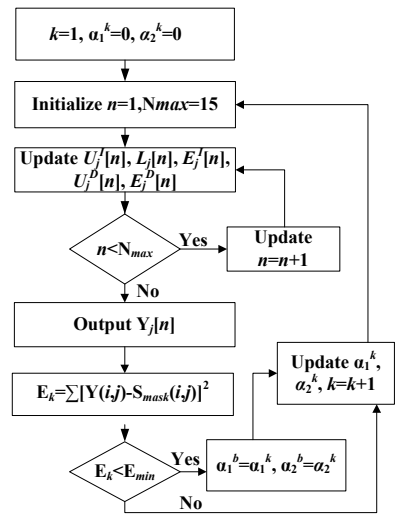

Figure 3. Optimization algorithm flow diagrams of parameters of neural network model 
Set different values of $\alpha_{1}{ }^{k}$ and $\alpha_{2}{ }^{k}$ (where the range of $\alpha_{1}$ and $\alpha_{2}$ is $[0,1]$ ), calculate the error between based on neural network model segmentation results and binarization mask image. When the error is minimum, the current value of $\alpha_{1}$ and $\alpha_{2}$ is regarded as the optimal solution $\alpha_{1}^{b}$ and $\alpha_{2}^{b}$ of parameters of $\alpha_{1}$ and $\alpha_{2}$ of the neural network model in current environment. Error function is given by:

$$
E_{k}=\sum_{\alpha_{1}^{k}, \alpha_{2}^{k}}\left[Y(i, j)-S_{m a s k}(i, j)\right]^{2}
$$

where $Y$ is segmentation results based on designed neural network model, $S_{\text {mask }}$ is binarization mask image. For solving the optimization problem, this paper uses the exhaustive search method, in which the parameters $\alpha_{1}{ }^{k}$ and $\alpha_{2}{ }^{k}$ update in step length 0.01 .Then constantly calculate error to find the minimum. Obtain the optimal solutions of parameters $\alpha_{1}$ and $\alpha_{2}$ of this model, which are best fit to the 36 calibration graphs. Make statistics for the distributions of $\alpha_{1}$ and $\alpha_{2}$, draw the curves and then using the Gaussian

\section{RESUlTS AND ANALYSIS}

\section{A. Calibration Graph Design and Acquisition}

In order to reflect the change characteristics of the different targets in underwater environment as far as possible, the calibration graphs are designed mainly from the aspects of different colors (including nine colors: red, orange, yellow, green, blue, blue, purple, black and multicolor ) and different shapes (rectangular, circular, triangle and Gaussian shape).Finally, we designed 36 calibration graphs. The polarization images of calibration graphs are collected under the condition of natural light, water depth is $50 \mathrm{~cm}$, and the distance between camera and target is $50 \mathrm{~cm}$. Three polarization images of calibration graphs were collected. They are respectively 0 degrees, 45 degrees and 90 degrees polarization image. 0 degree underwater polarization images of calibration graphs are shown in Fig. 4 (for example, four shapes' polarization images of purple calibration graphs).

\section{B. Optimization Results of Parameters of Neural Network Model}

According to the calculation process shown in Fig. 3, calculate the optimal solutions of parameters $\alpha_{1}$ and $\alpha_{2}$ of the neural network model, which are suitable for the 36 calibration graphs. Make statistics for the distribution of $\alpha_{1}$ and $\alpha_{2}$ with the spacing 0.1 . The statistical results are shown in Table I.

According to TABLE I, draw the distribution curves of $\alpha_{1}$ and $\alpha_{2}$, then use the Gaussian function to fitting. The results are shown in Fig. 5.

From Fig. 5(a) and Fig. 5(b), we can conclude that the parameters of this model have different optimal solutions for different image information, and present certain regularity.

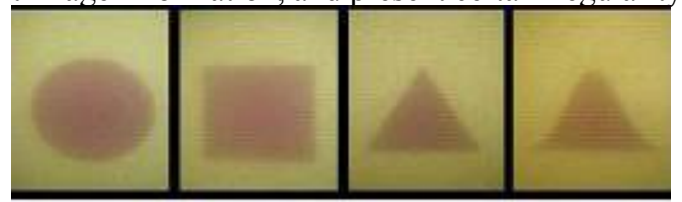

Figure 4. 0 degree polarization image of calibration underwater
TABLE I. STATISTICAL RESULTS

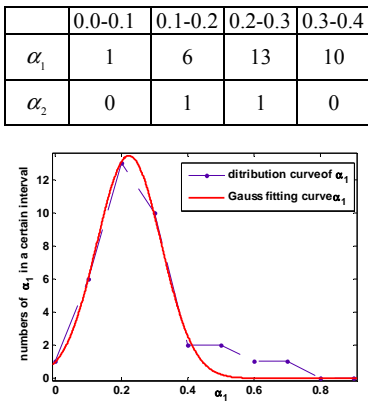

(a)

\begin{tabular}{|c|c|c|c|c|c|}
\hline $4-0.5$ & $0.5-0.6$ & $0.6-0.7$ & $0.7-0.8$ & $0.8-0.9$ & $0.9-1$ \\
\hline 2 & 2 & 1 & 1 & 0 & 0 \\
\hline 3 & 4 & 6 & 16 & 4 & 1 \\
\hline
\end{tabular}

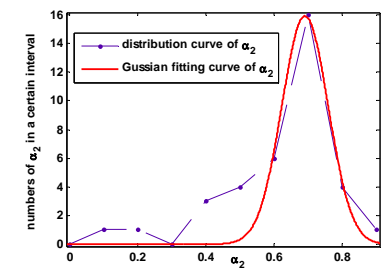

(b)
Figure 5. Distribution curves and Gaussian fitting curves:(a) distribution curve and Gaussian fitting curve of $\alpha_{1}$; (b) distribution curve and Gaussian fitting curve of $\alpha_{2}$

The range of parameter $\alpha_{1}$ is $[0.1,0.3]$ for the synthesis intensity images that including low frequency information. While the range of parameter $\alpha_{2}$ is $[0.6,0.8]$ for the polarization degree images that including high frequency information. The symmetry axis of Gaussian fitting curve is respectively $b_{1}=0.225, b_{2}=0.69$. Thus, the optimal solutions of parameters $\alpha_{1}$ and $\alpha_{2}$ of neural network model is 0.225 and 0.69.The result shows that the parameters of designed neural network model can adjust itself according to the characteristics of the input information, so that make the neural network better handle the current environment information, improving the accuracy of underwater image segmentation.

\section{Results of Simulation and Analysis}

This paper conducts simulation for different depth of underwater images to prove the advantages of the proposed algorithm in underwater image segmentation. And the result of image segmentation is compared with other image segmentation methods. Comparative experiment design is that employing Ostu and K-mean methods to carry on enhanced image segmentation. The enhanced image can be achieved by Dark Channel Prior method, which was proposed by reference [8]. The experimental results are shown in Fig. 6 and Fig. 7.

From Fig. 6(f), Fig. 6(g) and Fig. 6(h), whether using the proposed algorithm or using the Ostu and K-mean method with enhanced image, they can accurately locate the boundary of targets and separate the target from the background. From Fig. 7(f), Fig. 7(g) and Fig. 7(h), the segmentation results exit serious over-segmentation and can't separate the target from the background when used Ostu or K-mean method with enhanced image; while the proposed algorithm achieved a significant improvement, separated the target from the background, but the result still exits incomplete segmentation and some incorrect segmentation points. In summary, the designed neural network model can improve the accuracy of underwater image segmentation. 


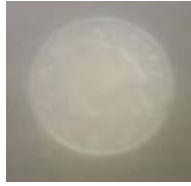

(a)

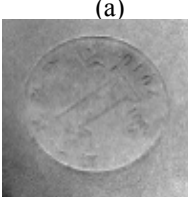

(e)
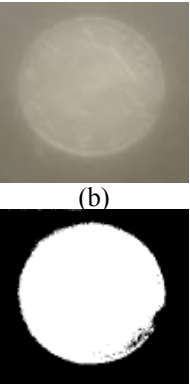

(f)
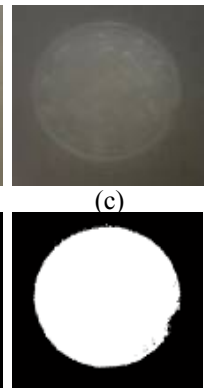

(g)
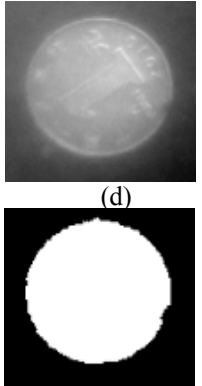

(h)
Figure 6. Image segmentation results of depth $20 \mathrm{~cm}$ of water: (a) 0 degree polarization image; (b) 45 degree polarization image; (c) 90 degree polarization image; (d) image of total intensity; (e) image of degree of polarization; (f) result of proposed algorithm; (g) enhanced image segmentation result of Ostu method; (h) enhanced image segmentation result of $\mathrm{K}$-mean method

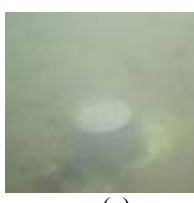

(a)

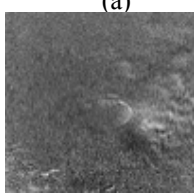

(e)

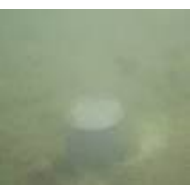

(b)

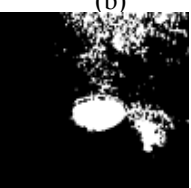

(f)

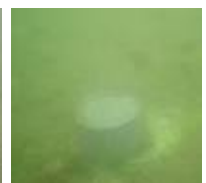

(c)

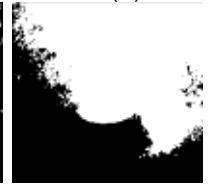

(g)

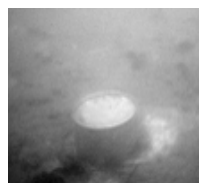

(d)

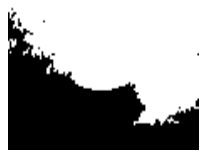

(h)
Figure 7. Image segmentation results of depth $50 \mathrm{~cm}$ of water: (a) 0 degree polarization image; (b) 45 degree polarization image; (c) 90 degree polarization image; (d) image of total intensity; (e) image of degree of polarization; (f) result of proposed algorithm; (g) enhanced image segmentation result of Ostu method; (h) enhanced image segmentation result of K-mean method

In order to evaluate the quality of image segmentation results, this paper exploits the overlap rate to describe the accuracy of image segmentation. Overlap rate is the ratio of segmentation result using custom method to expert manual segmentation result. The nearer overlap rate approaches to 1 , the higher segmentation accuracy of the method. It is also given by:

$$
P=\frac{\sum p_{i n}}{\sum p}
$$

where $p_{i n}$ is the number of pixels that the pixel value equal between segmentation result using custom method and experts manual segmentation result, $p$ is the number pixels of whole image. The overlap rate of segmentation results are given by TABLE II through the proposed algorithm, Ostu method and K- mean segmentation method with enhanced image.

From TABLE II, when the depth of water is shallow, the accuracy of image segmentation can reach more than $95 \%$ through the proposed algorithm, Ostu method and K-mean method with enhanced image. But with the increasing depth of water, the accuracy of image segmentation has a sharp decline through the Ostu method or K- mean method. The results show that they can't separate the target from the background. While the accuracy of segmentation using the proposed algorithm still reaches $90 \%$.

TABLE II. SEGMENTATION RESULTS EVALUATION

\begin{tabular}{|c|c|c|c|}
\hline $\begin{array}{c}\text { Segmentation } \\
\text { method }\end{array}$ & Overlap rate & $\begin{array}{c}\text { Segmentation } \\
\text { method }\end{array}$ & $\begin{array}{c}\text { Overlap } \\
\text { rate }\end{array}$ \\
\hline Fig. 6(f) & $98.34 \%$ & Fig. 7(f) & $90.00 \%$ \\
\hline Fig. 6(g) & $97.62 \%$ & Fig. 7(g) & $47.10 \%$ \\
\hline Fig.6(h) & $98.80 \%$ & Fig. 7(h) & $41.31 \%$ \\
\hline
\end{tabular}

\section{CONCLUSIONS}

Inspired by the information processing mechanism of mantis shrimps, this paper designed a feedback neural network model, in which the parameters are optimized using machine learning method. Based on the model, this paper constructed a frame of underwater polarization image segmentation. We could exploit the frame to combine the polarization and intensity information to achieve the underwater polarization image segmentation. Simulation results prove that the proposed feedback neural network model can improve the accuracy of underwater image segmentation.

\section{REFERENCES}

[1] John Y. Chiang and Ying Ching Chen, "Underwater Image Enhancement by Wavelength Compensation and Dehazing," IEEE Transactions on Image Processing, vol. 4, pp. 1756-1769, April 2012.

[2] Thomas W. Cronin, Nadav Shashar, Roy L. Caldwell, Justin Marshall, Alexander G. Cheroske, and Tsyr-Huei Chiou "Polarization Vision and Its Role in Biological Signaling," Integr. Comp. Biol., vol. 43, pp. 549-558, January 2003.

[3] Thomas W. Cronin and Justin Marshall, "Parallel Processing and Image Analysis in the Eyes of Mantis Shrimps," Biol. Bull., vol. 200, pp. 177-183, April 2001.

[4] Huibin Wang, Zhe Chen, Tong Xi, Xin Wang, and Lizhong Xu, "A Bionic Motion Tracking Model Inspired by the Eye of Mantis Shrimps," Engineering and Industries (ICEI), pp. 1-6, Nov. $292011-$ Dec. 12011.

[5] Sonja Kleinlogel, N. Justin Marshall, Julia M. Horwood, and Mike F. Land "Neuroarchitecture of the Color and Polarization Vision System of the Stomatopod Haptosquilla," The Journal of Comparative Neurology, vol. 467, pp.326-342, 2003.

[6] G. Di Stefano, L. Iacino, and H.Schiff, "What the Mantis Shrimp's Eyes (Possibly) Tell its Raptorial Appendages," Biol. Cybern., vol. 63, pp. 393-401, April 1990.

[7] Unnikrishnan, R., Pantofaru, and C.,Hebert, M., "Toward Objective Evaluation of Image Segmentation Algorithms," IEEE Transactions on Pattern Analysis and Machine Intelligence, vol. 29, pp. 929-944, 2007.

[8] Hung Yu Yang, Pei Yin Chen, and Yeu Horng Shiau, "Low Complexity Underwater Image Enhancement Based on Dark Channel Prior," Innovations in Bio-inspired Computing and Applications (IBICA), 2011 Second International Conference, pp. 17-21, December 16-18, 2011. 